Chem. Ber. 119, 950-959 (1986)

\title{
Additionen von Benzvalen an Nitriloxide. Eine Synthese für Benzvalen-3-carbonitril
}

\author{
Manfred Christl ${ }^{* a}$, Brigitte Mattauch $^{a}$, Hermann Irngartinger ${ }^{b}$ \\ und Annette Goldmann ${ }^{b}$ \\ Institut fur Organische Chemie der Universität Wüzburga \\ Am Hubland, D-8700 Würzburg, und \\ Organisch-Chemisches Institut der Universităt Heidelberg ${ }^{b}$, \\ Im Neuenheimer Feld 270, D-6900 Heidelberg 1
}

Eingegangen am 26. Juli 1985

2,4,6-Trimethylbenzonitriloxid, Benzonitriloxid und Knallsăure ergaben mit Benzvalen (1) in hohen Ausbeuten die $\Delta^{2}$-Isoxazoline $2 \mathbf{a}-\mathbf{c}$. Durch Konkurrenzexperimente wurde das Verhaltnis der Geschwindigkeitskonstanten der Additionen von Benzonitriloxid an 1 und Norbormen $k_{\text {Benzvalen }} / k_{\text {Norbornen }} \approx 1$ bestimmt. Das Knallsäure-Addukt $2 \mathrm{c}$ ließ sich in der folgenden einfachen Reaktionssequenz in Benzvalen-3-carbonitril (4) umwandeln: Ringoffnung durch Natriummethoxid zum B-Hydroxynitril 3, dessen Tosylierung und schließlich Eliminierung von Toluolsulfonsäure mit Kalium-tert-butoxid. - Mit Hilfe der Röntgenstrukturanalyse wurden für das Bicyclo[1.1.0]butan-Gerust von $\mathbf{2 b}$ der Interplanarwinkel zu $111.8^{\circ}$ und die Länge der Zentralbindung zu $1.464 \dot{A}$ ermittelt.

Additions of Benzvalene to Nitrile Oxides.

A Synthesis for Benzvalene-3-carbonitrile

2,4,6-Trimethylbenzonitrile oxide, benzonitrile oxide, and fulminic acid undergo cycloaddition to benzvalene (1) to give the $\Delta^{2}$-isoxazolines $2 \mathrm{a}-\mathrm{c}$ in high yields. By means of competition experiments the ratio of the rate constants for the reactions of 1 and norbornene with benzonitrile oxide has been determined to be $k_{\text {benzvalene }} / k_{\text {norbornene }} \approx 1$. The adduct $2 \mathrm{c}$ of fulminic acid was transformed to benzvalene-3-carbonitrile (4) by the following simple sequence: ring opening by sodium methoxide to yield the $\beta$-hydroxycarbonitrile 3 , tosylation of 3 , and, finally, elimination of toluenesulfonic acid with potassium tert-butoxide. - From the $X$-ray structure analysis of $2 \mathrm{~b}$ the dihedral angle and the length of the central bond of the bicyclo[1.1.0]butane moiety have been determined to be $111.8^{\circ}$ and $1.464 \AA$, respectively.

Norbornen addiert sich viel rascher an 1,3-Dipole des Diazoniumbetain- und NitriliumbetainTyps als Cyclohexen. Bei Diazomethan, Phenylazid, Diphenylnitrilimin und Benzonitriloxid betragen die Konkurrenzkonstanten 50501), 57002), 2103) und 61004). Da Benzvalen (1) wie Norbornen über das Strukturelement des Cyclopentens verfügt, erwarteten wir glatt verlaufende Cycloadditionen mit obigen 1,3-Dipolen und fanden sie auch5,6). Hier berichten wir im Detail über die Umsetzungen von 1 mit Nitriloxiden.

(C) VCH Verlagsgesellschaft mbH, D-6940 Weinheim, 1986 $0009-2940 / 86 / 0303-0950 \$ 02.50 / 0$ 


\section{A. Addition von Benzo- und 2,4,6-Trimethylbenzonitriloxid sowie von Knallsäure an 1}

Das stabile 2,4,6-Trimethylbenzonitriloxid wurde in etherischem Benzvalen (1) gelost und erbrachte nach 15 Stunden bei $20^{\circ} \mathrm{C}$ mit $97 \%$ Ausbeute das $\Delta^{2}$-Isoxazolin 2a. Benzonitriloxid, aus Benzhydroximoylchlorid in Gegenwart von 1 durch Triethylamin

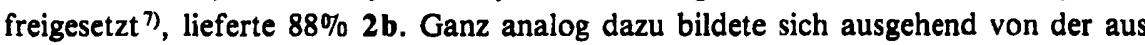
Formhydroximoyliodid erzeugten Knallsäure ${ }^{8)}$ das unsubstituierte Isoxazolin $2 \mathrm{c}$ mit $60 \%$ Ausbeute.

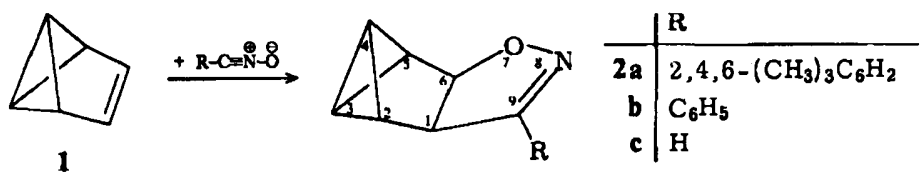

Die Struktur von 2b wird durch die Röntgenstrukturanalyse (Abschnitt D) belegt. In den ${ }^{1} \mathrm{H}$-NMR-Spektren findet sich der für $\Delta^{2}$-Isoxazoline typische ${ }^{9)}$ starke Unterschied zwischen den chemischen Verschiebungen von 1-H und 6-H $(\Delta \delta=1.19,1.30 \mathrm{ppm})$. Dagegen absorbieren 2-H und 5-H sowie 3-H und 4-H in einem verhältnismäßig engen Bereich $(\delta=2.0-2.6)$, und nur bei 2b und 2c konnten die betreffenden Signale aufgrund der charakteristischen Vicinalkopplung $J_{3,4} \approx 9 \mathrm{~Hz}$ wenigstens paarweise zugeordnet werden. Eine Unterscheidung innerhalb der Paare wäre durch Entkopplungsexperimente in einem Hochfeldspektrum möglich. Die ${ }^{13} \mathrm{C}$-chemischen Verschiebungen der Tricyclo[3.1.0.0 2,6] lich und of fenbaren so nur eine sehr geringe Fernwirkung der Phenylgruppe in $2 \mathbf{b}$. Lediglich am C-6-Signal von $\mathbf{2 b}$ tritt mit ca. $3 \mathrm{ppm}$ ein bemerkenswerter Tief feldeffekt auf, der vermutlich die durch die Heteroatome vermittelte Elektronenanziehung des Phenylrests signalisiert. Es gelang nicht, die Banden von C-2 und C-5 zu unterscheiden, weil die Kopplung dieser C-Atome mit 9-H in $2 \mathrm{c}$ nicht aufgelost ist. Dagegen sind die Banden von C-3 und C-4 jeweils spezifisch durch ihre Kopplungen mit 1-H und 6-H identifizierbar - ein Kriterium, das wir schon mehrfach angewandt haben ${ }^{10)}$. Gegenuber dem Spektrum des unsubstituierten Tricyclo[3.1.0.0.6.6 hexans treten an C-3 Tieffeldverschiebungen um 5.8 und $6.8 \mathrm{ppm}$ und an C-4 Hochfeldverschiebungen um 1.0 und $0.8 \mathrm{ppm}$ zutage. Damit ubt der anellierte $\Delta^{2}$-Isoxazolinring stärkere Effekte auf diese C-Atome aus als ein Cyclopentanring ${ }^{10 b}$ ). Im Rahmen des früher vorgeschlagenen Modells zur Deutung der Tieffeldverschiebungen spricht dies für eine gesteigerte Elektronenakzeptorqualităt des Heteroim Vergleich zum Homocyclus.

Kürzlich sind mit der Thermolyse von 3-substituierten 4-(Hydroxyimino)-5-oxo- $\Delta^{2}$-isoxazolinen ${ }^{11)}$ und der Hydrolyse von Trimethylsilancarbonitriloxid ${ }^{12)}$ zwei weitere Knallsäurequellen erschlossen und praparativ nutzbar gemacht worden. Neben der Cycloaddition von Knallsăure an Olefine führen auch die Oxidation von $\boldsymbol{N}$-unsubstituierten Isoxazolidinen mit Bleitetraacetat ${ }^{13 a)}$ und $\mathrm{N}$-Chlorsuccinimid ${ }^{13 b}$ ), die Einwirkung von Hydroxyharnstoff auf Crotonaldehyd mit anschließender Säure-Behandlung ${ }^{14)}$, die Anlagerung von (Trimethylsilyl)nitronaten an Olefine gefolgt von Trimethylsilanol-Eliminierung ${ }^{15)}$ und die Reduktion von 3-(Phenylsulfonyl)- $\Delta^{2}$-isoxazolinen mit Natriumborhydrid ${ }^{16}$ ) $\mathrm{zu}$ in 3-Stellung unsubstituierten $\Delta^{2}$-Isoxazolinen.

\section{B. Reaktivität von Benzvalen gegenüber Benzonitriloxid}

Nach Sustmann ${ }^{17)}$ ermöglicht die MO-Störungstheorie ein Verstăndnis der drei Typen von Dipolarophilen-Aktivitătsskalen, die von der Natur des jeweiligen 1,3-Dipols

Chem. Ber. 119 (1986) 
bestimmt werden. Zum Typ II gehören die Nitriloxide, denen gegenüber sowohl elektronenanziehende als auch elektronenliefernde Substituenten im Dipolarophil reaktionsfördernd wirken. Bei Olefinen mit einem energiereichen HOMO bestimmt dessen Wechselwirkung mit dem LUMO des 1,3-Dipols die Reaktivität. Dies dürfte für Norbornen gelten, da es über ein erstes Ionisationspotential von $8.97 \mathrm{eV}^{18)}$ verfügt. Seine Additionsgeschwindigkeit an Benzonitriloxid wird nur noch von der des $\beta$-Pyrrolidinostyrols ubertroffen ${ }^{4}$.

Das im Vergleich mit Norbornen kleinere erste Ionisationspotential von $8.55 \mathrm{eV}^{19}$ ) versprach für Benzvalen (1) eine höhere Reaktivität. In Analogie zu Lit. ") haben wir 1 und Norbornen um Benzonitriloxid konkurrieren lassen und folgendes Verhältnis der Geschwindigkeitskonstanten gefunden:

$$
\frac{k_{\text {Benzvalen }}}{k_{\text {Norbornen }}}=0.96
$$

Beide Olefine reagieren also praktisch gleich schnell. Rechnet man die höhere Symmetrie von 1 ein und den Befund, daß im Norbornen nur die exo-Seite angegriffen wird ${ }^{20)}$, so ergibt sich die doppelt so hohe Additionsgeschwindigkeit fur die exo-Seite der Norbornen-Doppelbindung wie für eine der beiden ăquivalenten Seiten der Benzvalen-Doppelbindung. Die Wirkung des energiereicheren HOMOs in 1 sowie die gunstigere sterische Situation $-7_{\text {syn }}-\mathrm{H}$ im Norbornen schirmt die Doppelbindung stärker $\mathrm{ab}$, als dies $1-\mathrm{H}(6-\mathrm{H})$ in 1 tut - werden somit durch den Faktor „ $\mathrm{X}^{\text {(1) }}$ des Norbornens überspielt, der auch für die exo-Selektivităt verantwortlich ist. Die Ursache des Faktors „X $\mathrm{X}^{\text {“ }}$ ist nach wie vor in der Diskussion. Houk et al. ${ }^{21)}$ fuhren sie auf die optimale antiperiplanare Anordnung der allylischen CC-Bindungen relativ zu den sich entwickelnden $\sigma$-Bindungen im Übergangszustand der Cycloaddition zurück, wăhrend SpangetLarsen und Gleiter ${ }^{22)}$ die hyperkonjugative Wechselwirkung des $\pi$-Orbitals mit den o-Orbitalen der $\mathrm{CH}_{2}$-Brücke verantwortlich machen. Im Hinblick auf die Spannungsenergie sollte zwischen den Reaktionen von 1 und Norbornen kein großer Unterschied bestehen. Aufgrund von Modellrechnungen nimmt sie im Norbornenfall im Zuge der Cycloaddition um ca. $5 \mathrm{kcal} / \mathrm{mol} \mathrm{ab}{ }^{11}$. Ein Betrag, der diesem Wert nahe kommen könnte, jedenfalls aber kleiner als $10 \mathrm{kcal} / \mathrm{mol}$ ist, läßt sich aus den Spannungsenergien von Benzvalen (1) und Bicyclo[1.1.0]butan abschätzen 6).

\section{Synthese von Benzvalen-3-carbonitril (4) aus $2 \mathrm{c}$}

In 3-Stellung unsubstituierte $\Delta^{2}$-Isoxazoline gehen unter der Einwirkung von Basen in $\beta$-Hydroxycarbonitrile aber $8,15 c, 23)$. Dieser Verbindungstyp entsteht auch bei der Decarboxylierung von $\Delta^{2}$-Isoxazolin-3-carbonsăuren 24), bei der Reduktion von 3-(Phenylsulfonyl)- $\Delta^{2}$ isoxazolinen mit Natriumamalgam ${ }^{25}$ ), bei der Einwirkung von Wasser auf 3-(Trimethylsilyl)- $\Delta^{2}$ isoxazoline 26) sowie bei der Addition von Cyanwasserstoff an Oxirane 27). Die formale Eliminierung von Wasser zu Acrylnitrilen wurde durch Acetylierung und dann Thermolyse ${ }^{28)}$ oder Erhitzen mit basischem Aluminiumoxid ${ }^{23)}$ erzielt.

Unter Anwendung dieser Reaktionsfolge haben wir aus 2c Benzvalen-3-carbonitril (4) synthetisiert. Die Behandlung von $2 \mathrm{c}$ mit Natriummethoxid erbrachte mit $39 \%$ Aus-

Chem. Ber. $119(1986)$ 
beute 3. Hinsichtlich seiner Umwandlung in 4 hielten wir wegen der zu erwartenden Thermolabilität von 4 die oben zitierten Bedingungen für ungeeignet, weshalb wir aus 3 das $p$-Toluolsulfonat bereiteten (44\% Ausbeute) und auf dieses Kalium-tert-butoxid einwirken ließen, woraus $4 \mathrm{mit}$ 46\% Ausbeute hervorging. Zwar erscheint die Gesamtausbeute uber die drei Schritte mit $8 \%$ als recht niedrig, jedoch sollte sie noch erheblich zu steigern sein, da bisher keinerlei Optimierung der Bedingungen vorgenommen wurde.

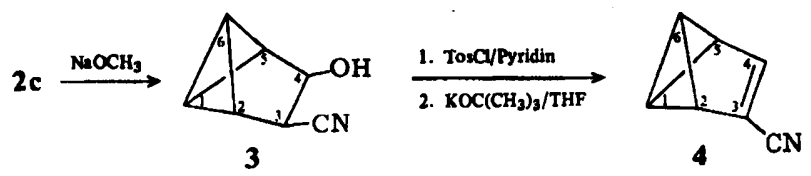

Beim Erhitzen auf $50^{\circ} \mathrm{C}$ in Hexadeuteriobenzol geht 4 mit einer Halbwertszeit von ca. elf Stunden in Benzonitril uber. Damit ist $\mathbf{4}$ thermisch etwas stabiler als das unsubstituierte Benzvalen (1), dessen Halbwertszeit bei $50^{\circ} \mathrm{C}$ sechs Stunden beträgt ${ }^{29)}$, und erheblich stabiler als 1-Methyl-30), 1-Brom- ${ }^{31)}$, 1-Chlor-31), 1-(Trimethylsilyl)- ${ }^{31)}$, 1-(Methoxycarbonyl)- ${ }^{31)}$, 1-( $p$-Tolylsulfonyl)- ${ }^{31)}$ und 3-Methylbenzvalen ${ }^{30)}$. Bei 3-Benzvalenylphenylsulfon und Bis(3-benzvalenyl)sulfon wurde hinsichtlich der Umlagerung in die Benzolreihe nichts mitgeteilt ${ }^{32)}$.

Benzvalen ${ }^{33)}$ und seine einfachen Derivate ${ }^{31,32)}$ neigen zur Detonation, weshalb wir auf eine sorgfältige Reinigung von 4 verzichteten, obwohl sich bei der Destillation und der sonstigen Handhabung von 20-mg-Mengen der kaum noch verunreinigten Substanz keine spontane Zersetzung ereignete.

Die cis-Anordnung der Substituenten in 3 folgt aus NMR-Kriterien ${ }^{10 c)}$ wie der Große der Kopplungskonstanten $J_{3,4}=6.8 \mathrm{~Hz}$ im ${ }^{1} \mathrm{H}$-NMR-Spektrum und der Feinstruktur der Signale von C-1 und C-6 im protonengekoppelten ${ }^{13} \mathrm{C}$-NMR-Spektrum. Typisch fur ein Benzvalenderivat absorbieren in $41-, 6-\mathrm{H}$ und $\mathrm{C}-1,6 \mathrm{mit} \delta=4.15$ bzw. 49.1 bei sehr tiefem Feld. Im gleichen Solvens $\left(\mathrm{CDCl}_{3}\right)$ liegen die entsprechenden Banden von 1 bei $\delta=3.85$ und 48.36,34a). Auf der Basis unseres Orbitalmodells zur Deutung der Benzvalen-Anomalie ${ }^{34)}$ muß man annehmen, daß zwar der Energieunterschied zwischen dem $\pi^{*}$-Orbital und dem besetzten $a_{2}$-Orbital des Bicyclo[1.1.0]butan-Systems in 4 kleiner ist als in 1, jedoch fallen auch die Koeffizienten des $\pi^{*}$-Orbitals von 4 wegen des ausgedehnteren $\pi$-Elektronensystems kleiner aus als in 1, womit das AusmaB der Wechselwirkung vergleichbar bleiben könnte.

\section{Kristall- und Molekularstruktur von $2 b$}

Im Rahmen unserer Untersuchungen über die Struktur von Bicyclo[1.1.0]butanDerivaten ${ }^{35)}$ und über die sterischen sowie durch Substituenten bedingten elektronischen Einflüsse auf die Brückenbindung ${ }^{36}$ ) in solchen Systemen haben wir die Kristallund Molekularstruktur von $\mathbf{2 b}$ mit Röntgenbeugung untersucht. Der Interplanarwinkel zwischen den beiden Dreiringen ist in definierter Weise durch die Verbrückung mit zwei C-Atomen fixiert. Da die Bruckenkopfatome Wasserstoffatome tragen, ist das Bindungssystem des Bicyclo[1.1.0]butan-Gerusts durch keine Substituenteneffekte beeinflußt.

Die Bindungslängen sind in $\mathrm{Abb} .1$ eingetragen, die auch die Atomnumerierung enthalt, wie sie in Abweichung von der systematischen Numerierung für die Struktur-

Chem. Ber. 119 (1986) 


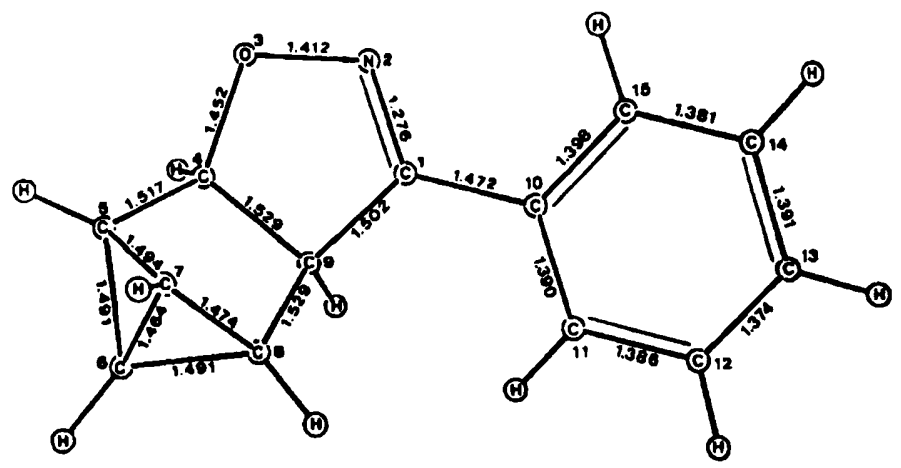

Abb. 1. Bindungslängen ( ) von $2 b$, Standardabweichungen $0.004-0.007 \AA$

analyse verwendet wurde. Die Bindungswinkel stehen in Tab. 1. Der Interplanarwinkel zwischen den beiden Dreiringen beträgt $111.8^{\circ}$, die Bindungslänge $\mathrm{C} 6-\mathrm{C} 7$ zwischen den Bruckenkopfatomen 1.464 Ả. Beide Werte passen sehr gut zu den Meßwerten anderer Bicyclo[1.1.0]butan-Derivate, aus denen sich eine direkte Abhängigkeit der Lănge der Brückenbindung vom Interplanarwinkel ergibt: Mit zunehmendem Interplanarwinkel nimmt diese Bindungslănge $\mathrm{zu}^{36,37}$. Im Vergleich $\mathrm{zu} \mathrm{C}-\mathrm{C}$-Bindungen in spannungsfreien Systemen ist auch in 2b dieser Bindungsabstand sehr kurz.

Tab. 1. Bindungswinkel ( ${ }^{\circ}$ ) von $\mathbf{2 b}$, Atomnumerierung siehe Abb. 1

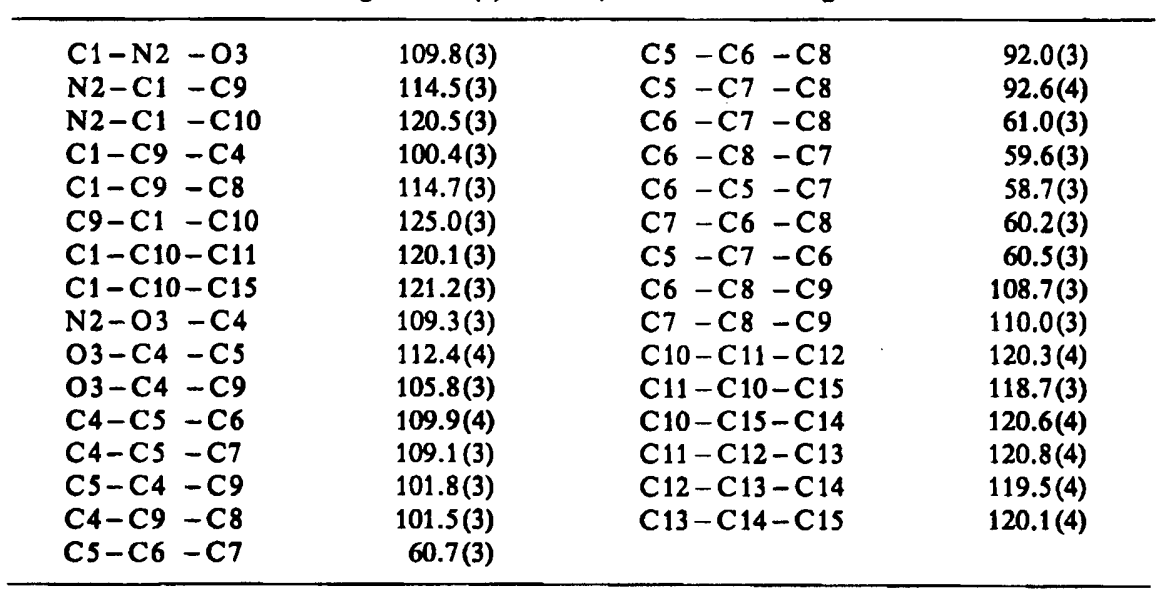

Die Ebene durch die Atome C4, C5, C8 und C9 bildet mit dem Isoxazolinring (C1, $\mathrm{N} 2, \mathrm{O3}, \mathrm{C4}, \mathrm{C9}$ ) einen Winkel von $117.3^{\circ}$. Dadurch erlangt das Brückenkopfatom $\mathrm{C7}$ kurze Kontakte zu C1, N2 und O3 (3.171(5), 3.455(5), 3.112(5) A). Der Phenylring weicht nur um $5.7^{\circ}$ von einer koplanaren Anordnung mit dem Isoxazolinring ab, so daß gute Konjugationsmoglichkeiten bestehen. Die Bindungslängen stehen damit in Einklang. 
Wir danken der Deutschen Forschungsgemeinschaft und dem Fonds der Chemischen Industrie für die Unterstutzung dieser Arbeit und Herrn Prof. Dr. W. Lüttke, Universităt Göttingen, für ein Protonen-gekoppeltes ${ }^{13} \mathrm{C}$-NMR-Spektrum von $2 \mathrm{~b}$.

\section{Experimenteller Teil}

${ }^{1}$ H-NMR: Varian A 60 und EM 390, Bruker WM 400. - ${ }^{13}$ C-NMR: Bruker WH 90 und WM 400. - IR: Beckman AccuLab 4. - UV: Zeiss RPQ 20C. - MS: AEI MS 902, Varian MAT CH 7. - Elementaranalysen: Carlo Erba Strumentatione Elemental Analyzer 1106. Molmasse: Mechrolab Dampfdruckosmometer 301 A. - Schmelzpunkte (nicht korrigiert): Mikroheiztisch der Fa. Reichert, Wien.

9-(2,4,6-Trimethylphenyl)-7-oxa-8-azatetracyclo[4.3.0.02.4.03.5Jnon-8-en (2a): $1.00 \mathrm{~g} \quad(6.20$ mmol) 2,4,6-Trimethylbenzonitriloxid ${ }^{38}$ ) wurden in $15 \mathrm{ml}$ etherischem Benzvalen (1) (9.0 mmol) aufgelost und $15 \mathrm{~h}$ bei Raumtemp. aufbewahrt. Nach dem Eindampfen hinterblieben $1.45 \mathrm{~g}$ (97\%) 2 a als farblose Kristalle mit Schmp. $96-100^{\circ} \mathrm{C}$, nach Umlosen aus Methanol/Wasser $101=103^{\circ} \mathrm{C}$. $-{ }^{1} \mathrm{H}-\mathrm{NMR}\left(\mathrm{CCl}_{4}\right): \delta=2.0-2.6$ (m; 2-, 3-, 4-, 5-H), 2.27 (s; $3 \mathrm{CH}_{3}$ ), 3.64 (br. d, $\left.J_{1,6}=8.5 \mathrm{~Hz} ; 1-\mathrm{H}\right), 4.94$ (br. d; 6-H), 6.78 (br. s; 2 aromat. H).

$\mathrm{C}_{16} \mathrm{H}_{17} \mathrm{NO}(239.3)$ Ber. C 80.30 H 7.16 N 5.85

Gef. C 80.04 H 7.14 N 6.00 Molmasse 236 (osmometr. in Benzol)

9-Phenyl-7-oxa-8-azatetracyclo[4.3.0.02,4.0.5.5non-8-en (2b): Dem Gemisch aus $20 \mathrm{ml}$ etherischem 1 (13.0 mmol) und $2.0 \mathrm{ml}(14.5 \mathrm{mmol})$ Triethylamin fugte man unter Eiskuhlung und Ruhren innerhalb von $5 \mathrm{~min}$ tropfenweise $1.56 \mathrm{~g}(10.0 \mathrm{mmol})$ Benzhydroximoylchlorid ${ }^{39)}$ in $10 \mathrm{ml}$ Ether zu. Man ruhrte $1 \mathrm{~h}$, extrahierte mit Wasser, trocknete und engte die Etherphase ein. Es hinterblieben $2.04 \mathrm{~g}$ bräunliche Kristalle, nach Umlosen aus Cyclohexan $1.27 \mathrm{~g} 2 \mathrm{~b}$ in farblosen Kristallen mit Schmp. $110-111^{\circ} \mathrm{C}$. Aus dem Mutterlaugenruckstand destillierten bei $140-160^{\circ} \mathrm{C}$ (Bad)/0.001 Torr weitere $0.47 \mathrm{~g}$ reines $2 \mathrm{~b}$ (Gesamtausb. 88\%). - UV (Cyclohexan): $\lambda_{\max }(\log \varepsilon)$ $=265$ (4.05), 271 (sh, 4.03), 280 (sh, 3.82), 285 (sh, 3.63), $291 \mathrm{~nm}$ (sh, 2.96). - MS (20 eV): $m / z=197\left(100 \%, \mathrm{M}^{+}\right), 196(26), 168(43), 167(17), 158(13), 119(35), 78(22), 66(22)$. ${ }^{1} \mathrm{H}-\mathrm{NMR}\left(\mathrm{CCl}_{4}\right): \delta=2.05$ und 2.20 (br. AB-Spektrum, $J_{3,4} \approx 9 \mathrm{~Hz} ; 3-, 4-\mathrm{H}$ ), 2.48 (br. s; 2-, 5-H), 3.79 (br. d, $\left.J_{1,6}=7.9 \mathrm{~Hz} ; 1-\mathrm{H}\right), 4.98$ (br. d; 6-H), $\mathrm{C}_{6} \mathrm{H}_{5}: 7.1-7.5(3 \mathrm{H}), 7.5-7.9(2 \mathrm{H})$. ${ }^{13} \mathrm{C}-\mathrm{NMR}\left(\mathrm{CDCl} \mathrm{C}_{3}\right): \delta=1.36\left(\mathrm{dtd},{ }^{1} J_{\mathrm{C}, \mathrm{H}}=218.8 \mathrm{~Hz},{ }^{3} J_{\mathrm{C}-4,1-\mathrm{H}} \approx{ }^{3} J_{\mathrm{C}-4,6-\mathrm{H}} \approx 7.8 \mathrm{~Hz},{ }^{2} J_{\mathrm{C}-4,3-\mathrm{H}}=\right.$ $3.8 \mathrm{~Hz} ; \mathrm{C}-4$ ), 8.24 (dddt, ${ }^{1} J_{\mathrm{C}, \mathrm{H}}=217.8 \mathrm{~Hz},{ }^{2} J_{\mathrm{C}-3,4-\mathrm{H}}=3.8 \mathrm{~Hz}$, weitere Kopplungen 1.2 und $2 \mathrm{mal} 0.8 \mathrm{~Hz}$; C-3), 37.68 (ddqd, ${ }^{1} J_{\mathrm{C}, \mathrm{H}}=170.3 \mathrm{~Hz},{ }^{3} J_{\mathrm{C}-2, \mathrm{~S}-\mathrm{H}}$ oder ${ }^{3} J_{\mathrm{C}-5,2-\mathrm{H}}=14.3 \mathrm{~Hz}$, weitere Kopplungen 3mal 4.1 und $1.9 \mathrm{~Hz} ; \mathrm{C}-2$ oder C-5), 39.79 ( $\approx$ ddquint, ${ }^{1} J_{\mathrm{C}, \mathrm{H}}=170.2 \mathrm{~Hz},{ }^{3} J_{\mathrm{C}-5,2-\mathrm{H}}$ oder ${ }^{3} J_{\mathrm{C}-2,5-\mathrm{H}}=13.3 \mathrm{~Hz}$, weitere Kopplungen $4 \mathrm{mal} \approx 4.1 \mathrm{~Hz} ; \mathrm{C}-5$ oder C-2), 56.07 (dt, ${ }^{1} J_{\mathrm{C}, \mathrm{H}}=142.5 \mathrm{~Hz}$, weitere Kopplungen $\left.2 \mathrm{mal} 5.6 \mathrm{~Hz} ; \mathrm{C}-1\right), 88.74\left(\mathrm{dtd},{ }^{1} J_{\mathrm{C}, \mathrm{H}}=158.0 \mathrm{~Hz}\right.$, weitere Kopplungen $2 \mathrm{mal} 5.5$ und $0.7 \mathrm{~Hz}$; C-6), 156.09 (s; C-9), $\mathrm{C}_{6} \mathrm{H}_{5}: 126.21$ und 128.38 (o-C, $m-\mathrm{C}$ ), 129.47 ( $p$-C), 129.60 (ipso-C).

$$
\mathrm{C}_{13} \mathrm{H}_{11} \mathrm{NO}(197.2) \text { Ber. C } 79.17 \text { H } 5.62 \text { N 7.10 Gef. C } 79.28 \text { H } 5.75 \text { N } 7.14
$$

7-Oxa-8-azatetracyclo/4.3.0.0.0.4. $0^{3,5}$ Jnon-8-en (2c): Unter Stickstoff gab man zu $1.56 \mathrm{~g}$ (20.0 mmol) 1 und $1.00 \mathrm{~g} \mathrm{NaHCO}_{3}$ in $45 \mathrm{ml}$ Ether bei $0^{\circ} \mathrm{C}$ in $1 \mathrm{~min} 3.22 \mathrm{~g} \mathrm{(18.8} \mathrm{mmol)} \mathrm{Form-}$ hydroximoyliodid $\left.{ }^{8}\right)$ in $20 \mathrm{ml}$ Ether. Unter Ruhren tropfte man bei $0^{\circ} \mathrm{C} 3.03 \mathrm{~g}(30.0 \mathrm{mmol}) \mathrm{Tri}-$ ethylamin in $15 \mathrm{ml}$ Ether innerhalb von $2 \mathrm{~h}$ zu und ließ dann auf $20^{\circ} \mathrm{C}$ kommen. Man gab dann soviel Wasser zu, daß sich der Niederschlag loste, extrahierte die waßßrige Phase mit Ether und die vereinigten etherischen Phasen mit Wasser, trocknete uber $\mathrm{Na}_{2} \mathrm{SO}_{4}$ und engte i. Vak. ein. Aus dem Rückstand destillierten bei $60-80^{\circ} \mathrm{C}(\mathrm{Bad}) / 0.1$ Torr $1.36 \mathrm{~g}(60 \%) 2 \mathrm{c}$ als gelbe, nach Redestillation farblose Flussigkeit, die sich bei $20^{\circ} \mathrm{C}$ innerhalb von Stunden nach Schwarz verfärbte. - IR ( $\left.\mathrm{CCl}_{4}\right): 3065$ (m), 2960 (s), $1593(\mathrm{~m}, \mathrm{C}=\mathrm{N}), 1400$ (w), 1318 (m), 1288 (m), 1275 (m),

Chem. Ber. 119 (1986) 
$1242(\mathrm{~m}), 1148(\mathrm{~m}), 1123(\mathrm{~s}), 1035(\mathrm{~m}), 968(\mathrm{~s}), 925(\mathrm{~m}), 893(\mathrm{~s}), 850 \mathrm{~cm}^{-1}(\mathrm{~s}) .-\mathrm{MS}(70 \mathrm{eV})$ : $m / z=121\left(3 \%, M^{+}\right), 92(15), 78(45), 66(32), 65(26), 52(100), 51(16), 50(11), 40(13)$, 39 (76). $-{ }^{1} \mathrm{H}-\mathrm{NMR}\left(\mathrm{CDCl}_{3}\right): \delta=2.08$ und 2.22 (br. AB-Spektrum, $\left.J_{3,4}=9 \mathrm{~Hz} ; 3 \cdot \mathrm{H}, 4-\mathrm{H}\right)$, 2.43 (br. s; 2-, 5-H), 3.57 (br. d, $J_{1,6}=8.4 \mathrm{~Hz} ; 1-\mathrm{H}$ ), 4.87 (br. d; 6-H), 6.88 (br. s; 9-H). ${ }^{13} \mathrm{C}-\mathrm{NMR}\left(\mathrm{CDCl}_{3}\right)$ : Die Feinstrukturen der Signale sind weitgehend die gleichen wie im Spektrum von $2 \mathrm{~b}$. Lediglich in den Banden von C-1 und C-6 taucht eine zusătzliche Kopplung, nămlich jene zu 9-H, auf; $\delta=1.2(218.4 \mathrm{~Hz} ; \mathrm{C}-4), 7.2(216.9 \mathrm{~Hz}$; C-3), 37.2 und 39.4 (169.9 bzw. $169.1 \mathrm{~Hz}$ C-2 und C-5), $57.5\left(144.1 \mathrm{~Hz},{ }^{2} J_{\mathrm{C}-1,9-\mathrm{H}}=10.3 \mathrm{~Hz} ; \mathrm{C}-1\right), 85.8\left(158.8 \mathrm{~Hz},{ }^{3} J_{\mathrm{C}-6,9-\mathrm{H}}=4.7 \mathrm{~Hz}\right.$; C-6), $146.5(186.0 \mathrm{~Hz}$; C-9).

\section{$\mathrm{C}_{7} \mathrm{H}_{7} \mathrm{NO}$ (121.1) Ber. C 69.40 H 5.82 N 11.56 Gef. C 69.08 H 5.94 N 10.82}

Konkurrenzversuche zur Ermittlung der relativen Reaktivitat von Benzvalen (1) und Norbornen gegenuber Benzonitriloxid: Man legte die beiden Olefine in der 10-15fachen Menge absol. Ethers vor und bestimmte ihre Konzentration durch ein ${ }^{1} \mathrm{H}-\mathrm{NMR}$-Spektrum anhand der Banden bei $\delta=5.87$ (1), 5.97 (Norbornen) und 6.87 (interner Standard Mesitylen). Nach der Zugabe von Benzhydroximoylchlorid kühlte man unter Stickstoff auf $0^{\circ} \mathrm{C}$ und setzte unter Rühren durch $\mathrm{Zu}$ tropfen etherischen Triethylamins innerhalb von $1.5 \mathrm{~h}$ Benzonitriloxid frei. Man rührte 1 weitere $\mathrm{h}$ bei $0^{\circ} \mathrm{C}, 2 \mathrm{~h}$ bei $20^{\circ} \mathrm{C}$ und arbeitete wie bei der Darstellung von $2 \mathrm{~b}$ auf. Die Zusammensetzung des Rohprodukts, bestehend aus $\mathbf{2 b}$ und dem entsprechenden Norbornen-Addukt, wurde durch ein ${ }^{1} \mathrm{H}$-NMR-Spektrum in $\mathrm{CDCl}_{3}$ mit Mesitylen als internem Standard anhand der Banden der Protonen an den $\Delta^{2}$-Isoxazolinringen ermittelt. Die Konkurrenzkonstante wurde wie in Lit.4) berechnet. In der folgenden Tabelle sind Ansatzgrößen und Konkurrenzkonstanten der einzelnen Versuche zusammengestellt.

\begin{tabular}{cccccccc}
\hline $\begin{array}{c}\text { Vers. } \\
\text { Nr. }\end{array}$ & $\begin{array}{c}\text { mmol } \\
\text { Benz- } \\
\text { hydroxi- } \\
\text { moylchlorid }\end{array}$ & $\begin{array}{c}\text { mmol } \\
\text { Benz- } \\
\text { valen } \\
\text { (1) }\end{array}$ & $\begin{array}{c}\text { mmol } \\
\text { Norbor- } \\
\text { nen }\end{array}$ & $\begin{array}{c}\text { mmol } \\
\text { Benzvalen- } \\
\text { Addukt } \\
\text { (2b) }\end{array}$ & $\begin{array}{c}\text { mmol } \\
\text { Norbor- } \\
\text { nen- } \\
\text { Addukt }\end{array}$ & $\begin{array}{c}\text { Konkur- } \\
\text { renz- } \\
\text { konstante }\end{array}$ & $\begin{array}{c}\% \text { Ge- } \\
\text { samt- } \\
\text { ausbeute }\end{array}$ \\
\hline 1 & 5.01 & 17.29 & 9.66 & 2.50 & 1.54 & 0.91 & 81 \\
2 & $\mathbf{8 . 0 3}$ & 11.75 & 12.63 & 3.12 & 3.42 & 1.00 & 82 \\
3 & 5.01 & 7.72 & 14.65 & 1.43 & 2.79 & 0.97 & 84
\end{tabular}

cis-4-Hydroxytricyclo/3.1.0.0.0.6 Jhexan-3-carbonitril (3): Die geruhrte Losung von $615 \mathrm{mg}$ $(5.08 \mathrm{mmol}) 2 \mathrm{c}$ in $3 \mathrm{ml}$ absol. Methanol wurde bei $25^{\circ} \mathrm{C}$ innerhalb von $3 \mathrm{~min}$ mit $540 \mathrm{mg}$ $(10.0 \mathrm{mmol})$ Natriummethoxid in $5 \mathrm{ml}$ absol. Methanol versetzt, wobei sich das Gemisch leicht erwärmte und nach Braun verfärbte. Man ruhrte 1 h bei $25^{\circ} \mathrm{C}$, setzte soviel Wasser und Ether zu, daß sich 2 Phasen bildeten, trocknete die Etherphase über $\mathrm{Na}_{2} \mathrm{SO}_{4}$ und engte i. Vak. ein. Aus dem Ruckstand (351 mg braunes OOl) destillierten bei $60-80^{\circ} \mathrm{C}(\mathrm{Bad}) / 10^{-3}$ Torr $240 \mathrm{mg}(39 \%) 3$ als gelbliches $O l$, aus dem nach Zusatz von wenig Ether innerhalb einiger Wochen $165 \mathrm{mg}$ (27\%) farblose Kristalle mit Schmp. $48-50^{\circ} \mathrm{C}$ fielen. - IR (KBr): 3300 (breit, OH), 3125, 3050, 2955, 2930 (jeweils $C-H), 2248 \mathrm{~cm}^{-1}(C=N)$. - MS $(70 \mathrm{eV}): m / z=121\left(6 \%, \mathrm{M}^{+}\right), 93(14), 92(15)$, 67 (18), 66 (100), 65 (30), 53 (11), 52 (62), 51 (18), 50 (13), 40 (11), 39 (31). - 'H-NMR (CDCl $)$ : $\delta=2.17$ (dtd, $J_{1,6}=8.5 \mathrm{~Hz}, J_{1,2}=J_{1,5}=2.0 \mathrm{~Hz}, J_{1,3}=0.8 \mathrm{~Hz} ; 1-\mathrm{H}$ ), 2.39 und 2.45 (jeweils $\mathrm{dm}, J_{2,5} \approx 5 \mathrm{~Hz} ; 2-\mathrm{H}, 5-\mathrm{H}$ ), 2.47 (dquint, teilweise überlagert, $J_{2,6} \approx J_{3,6} \approx J_{4,6} \approx J_{5,6}$ $=1.5 \mathrm{~Hz} ; 6-\mathrm{H}), 2.84\left(\approx \mathrm{dq}, J_{3,4}=6.8 \mathrm{~Hz}, J_{2,3} \approx 1.2 \mathrm{~Hz} ; 3-\mathrm{H}\right), 3.09\left(\mathrm{~d}, J_{4, \mathrm{OH}}=6.8 \mathrm{~Hz} ; \mathrm{OH}\right)$, $4.34\left(\mathrm{tt}, J_{4,5} \approx 1.5 \mathrm{~Hz} ; 4-\mathrm{H}\right) .{ }^{13} \mathrm{C}-\mathrm{NMR}\left(\mathrm{CDCl} \mathrm{C}_{3}\right): \delta=3.18\left(\mathrm{dtd},{ }^{1} J_{\mathrm{C}, \mathrm{H}}=220 \mathrm{~Hz},{ }^{3} J_{\mathrm{C}-6,3-\mathrm{H}}=\right.$ $\left.{ }^{3} J_{\mathrm{C}-6,4-\mathrm{H}}=8 \mathrm{~Hz},{ }^{2} J_{\mathrm{C}-6,1-\mathrm{H}}=3 \mathrm{~Hz} ; \mathrm{C}-6\right), 4.94\left(\mathrm{~d},{ }^{1} J_{\mathrm{C}, \mathrm{H}}=219 \mathrm{~Hz} ; \mathrm{C}-1\right), 35.68$ und 39.99 (jeweils $\left.\mathrm{ddm},{ }^{1} J_{\mathrm{C}, \mathrm{H}}=174 \mathrm{bzw} .170 \mathrm{~Hz},{ }^{3} J_{\mathrm{C}-2,5-\mathrm{H}} \approx{ }^{3} J_{\mathrm{C}-5,2-\mathrm{H}} \approx 13 \mathrm{~Hz} ; \mathrm{C}-2, \mathrm{C}-5\right), 38.02$ (dtd, ${ }^{1} J_{\mathrm{C}, \mathrm{H}}=$ $142 \mathrm{~Hz}$, weitere Linienabstände 7 und $3 \mathrm{~Hz} ; \mathrm{C}-3), 73.60\left(\mathrm{dtd},{ }^{1} J_{\mathrm{C}, \mathrm{H}}=156 \mathrm{~Hz}\right.$, weitere Linienabstände 7 und $3 \mathrm{~Hz} ; \mathrm{C}-4), 118.68\left(\mathrm{~d},{ }^{2} J_{\mathrm{CN}, 3-\mathrm{H}}(\right.$ ?) $=8 \mathrm{~Hz} ; \mathrm{CN})$.

$\mathrm{C}_{7} \mathrm{H}_{7} \mathrm{NO}(121.1)$ Ber. C 69.40 H 5.82 N 11.56 Gef. C 69.36 H 5.82 N 11.60

Chem. Ber. 119 (1986) 
p-Toluolsulfonat von 3: Zur eisgekühlten gerührten Losung von $115 \mathrm{mg}(0.95 \mathrm{mmol}) 3$ in $2 \mathrm{ml}$ absol. Pyridin gab man portionsweise $210 \mathrm{mg}(1.10 \mathrm{mmol})$ p-Toluolsulfonylchlorid. Man ruhrte $5 \mathrm{~h}$ bei $0^{\circ} \mathrm{C}$, goß dann das Gemisch auf $5 \mathrm{ml}$ Eis/Wasser, extrahierte mehrmals mit Ether, trocknete über $\mathrm{Na}_{2} \mathrm{SO}_{4}$ und engte i. Vak. ein. Aus dem zuruckbleibenden braunen $\mathrm{Ol}(160 \mathrm{mg})$ gewann man mit Methanol/Hexan $115 \mathrm{mg}(44 \%)$ beige Kristalle mit Schmp. $55-58^{\circ} \mathrm{C}$. - IR (KBr): $2250(C=N), 1600 \mathrm{~cm}^{-1}$ (aromat. $\left.C=C\right) .-M S(70 \mathrm{eV}): m / z=275\left(2 \%, \mathrm{M}^{+}\right), 155(41)$, 120 (16), 108 (10), 104 (19), 103 (11), 92 (23), 91 (100), 79 (10), 77 (27), 66 (14), 65 (41), 57 (14), 56 (11), 53 (14), 52 (15), 51 (15), 43 (12), 41 (17), 39 (26). - ${ }^{1} \mathrm{H}-\mathrm{NMR}\left(\mathrm{CDCl}_{3}\right): \delta=2.15$ (s; $\mathrm{CH}_{3}$ ), 2.32 und 2.60 (jeweils $\mathrm{dm}, J_{1,6}=9 \mathrm{~Hz} ; 1-, 6-\mathrm{H}$ ), 2.47 (m; 2-, 5-H), 2.92 (br. d, $J_{3,4}=$ $7.5 \mathrm{~Hz}$; 3-H), 4.92 (br. d; 4-H), 7.38 und 7.91 (AA'BB'-Spektrum; aromat. $\mathrm{H}$ ).

$\mathrm{C}_{14} \mathrm{H}_{13} \mathrm{NO}_{3} \mathrm{~S}$ (275.3) Ber. C $61.07 \mathrm{H} 4.76$ N 5.09 Gef. C $61.86 \mathrm{H} 4.98 \mathrm{~N} 5.42$

Tricyclo[3.1.0.02.6 Jhex-3-en-3-carbonitril (Benzvalen-3-carbonitril, 4): $\mathrm{Zu} 400 \mathrm{mg}$ (1.45 mmol) des $p$-Toluolsulfonats von 3 in $5 \mathrm{ml}$ absol. Tetrahydrofuran tropfte man unter Ruhren innerhalb von $5 \mathrm{~min} 185 \mathrm{mg}(1.65 \mathrm{mmol})$ Kalium-tert-butoxid in $2 \mathrm{ml}$ Tetrahydrofuran. Es bildete sich rasch ein Niederschlag (Kalium-p-toluolsulfonat); nach $2.5 \mathrm{~h}$ filtrierte man und engte das Filtrat bei $0^{\circ} \mathrm{C} / 40$ Torr bis auf $0.5 \mathrm{ml}$ ein. Aus Sicherheitsgründen fügte man zur Bereitung der NMR-Probe $3 \mathrm{ml} \mathrm{CDCl} \mathrm{zu}_{3}$ und engte erneut bis auf $0.5 \mathrm{ml}$ ein. Laut Integration im ${ }^{1} \mathrm{H}-\mathrm{NMR}$ Spektrum mit Dimethylformamid als internem Standard lagen $69 \mathrm{mg}(46 \%) 4$ vor. Die Destillation einer $24.7 \mathrm{mg} 4$ enthaltenden Probe bei $40-50^{\circ} \mathrm{C}$ (Bad)/0.1 Torr erbrachte $21.5 \mathrm{mg}$ kaum noch verunreinigtes 4 als farblose Flussigkeit. - IR $\left(\mathrm{CCl}_{4}\right): 3080,2988,2944$ (jeweils $\left.\mathrm{C}-\mathrm{H}\right)$, $2222(C=N), 1578 \mathrm{~cm}^{-1}(C=C)$. $-M S(70 \mathrm{eV}): m / z=103\left(100 \%, \mathrm{M}^{+}\right), 77(6), 76(36), 75(8)$, $66(8), 51$ (9), $50(15), 39(6) .-{ }^{1} \mathrm{H}-\mathrm{NMR}\left(\mathrm{CDCl}_{3}\right): \delta=2.28$ und 2.44 (jeweils dq, $J_{2,3}=6.2 \mathrm{~Hz}$; $2-, 5-\mathrm{H}), 4.15\left(\mathrm{t}, J_{1,2}=J_{1,5}=1.5 \mathrm{~Hz} ; 1-, 6-\mathrm{H}\right), 6.85\left(\mathrm{dd}, J_{2,4}\right.$ und $\left.J_{4,5}=1.5,1.9 \mathrm{~Hz} ; 4-\mathrm{H}\right)$. ${ }^{13} \mathrm{C}-\mathrm{NMR}\left(\mathrm{CDCl}_{3}\right): \delta=38.54$ (d, $176 \mathrm{~Hz}$, Doppelsignal; C-2, -5), 49.10 (d, $213 \mathrm{~Hz}$; C-1, -6), 116.07 und 116.92 (jeweils s; C-3, CN), 152.10 (d, $178 \mathrm{~Hz}$; C-4).

Zur Thermolyse von 4 loste man einige $\mathrm{mg}$ der destillierten Substanz in $\mathrm{C}_{6} \mathrm{D}_{6}$, erhitzte im NMR-Röhrchen auf $50^{\circ} \mathrm{C}$, nahm von Zeit zu Zeit ein Spektrum auf und errechnete aus den Integralen der Signale von 4 und dem gebildeten Benzonitril (Identifizierung durch Vergleich der Signalform mit der der authentischen Substanz) die Halbwertszeit zu ca. $11 \mathrm{~h}$.

Tab. 2. Atomkoordinaten und thermische Parameter von 2 b, Atomnumerierung siehe Abb. 1, $\mathrm{UEQU}=\frac{1}{3} \sum_{j} U_{i j} a_{i}^{*} a_{j}^{*} \mathbf{a}_{i} \cdot \mathbf{a}_{j}$

\begin{tabular}{lrrrr}
\hline Atom & $X / A$ & $Y / B$ & $Z / C$ & UEQU \\
\hline C1 & $1979(3)$ & $2997(2)$ & $7843(4)$ & $367(17)$ \\
N2 & $2012(3)$ & $2157(2)$ & $6936(4)$ & $520(17)$ \\
O3 & $918(3)$ & $1493(2)$ & $7321(4)$ & $643(17)$ \\
C4 & $103(4)$ & $2010(3)$ & $8585(5)$ & $520(20)$ \\
C5 & $-1234(4)$ & $2368(3)$ & $7904(6)$ & $610(27)$ \\
C6 & $-1620(4)$ & $3411(4)$ & $8670(5)$ & $370(23)$ \\
C7 & $-1059(4)$ & $3397(4)$ & $6979(6)$ & $600(23)$ \\
C8 & $-277(3)$ & $3875(3)$ & $8348(5)$ & $443(20)$ \\
C9 & $777(3)$ & $3082(3)$ & $8952(4)$ & $390(20)$ \\
C10 & $3064(3)$ & $3787(2)$ & $7767(4)$ & $363(13)$ \\
C11 & $2997(3)$ & $4717(3)$ & $8717(5)$ & $447(20)$ \\
C12 & $4032(4)$ & $5449(3)$ & $8684(6)$ & $543(23)$ \\
C13 & $5141(4)$ & $5269(3)$ & $7719(6)$ & $570(23)$ \\
C14 & $5215(4)$ & $4347(3)$ & $6747(5)$ & $530(20)$ \\
C15 & $4191(3)$ & $3611(3)$ & $3611(5)$ & $450(20)$ \\
\hline
\end{tabular}

Chem. Ber. 119 (1986) 
Rontgenstrukturanalyse von $2 \mathrm{~b}$ : Die Gitterkonstanten ergaben sich aus der Vermessung von 30 Reflexen hoher Ordnung $\left(2 \Theta>42^{\circ}\right): a=10.052(1) ; b=12.493(2) ; c=7.992(1) \dot{A} ;$ Raumgruppe: $P 2_{1} 2_{1} 2_{1} ; Z=4 ; D_{\text {ber }}=1.29 \mathrm{Mg} / \mathrm{m}^{3}$. Mit einem Kristall der Größe $0.22 \times 0.24$ $\times 0.36 \mathrm{~mm}^{3}$ wurden auf einem Diffraktometer (Siemens AED, Graphitmonochromator, $\omega-2 \Theta$ Abtastung, Mo- $K_{\alpha}$-Strahlung) bis $\sin \Theta / \lambda=0.664 \dot{A}^{-1} 1418$ unabhăngige Reflexe vermessen. Davon wurden 395 Reflexe als unbeobachtet angesehen $\left(F_{0}^{4}<5 \sigma^{2}\left(F_{0}^{2}\right)\right)$. Die Strukturlösung erfolgte nach Direkten Methoden mit dem Programm MULTAN40). Die Kleinste-QuadrateVerfeinerung im Voll-Matrix-Verfahren mit anisotropen Temperaturfaktoren für die $\mathrm{C}$-, $\mathrm{N}$ - und O-Atome und isotropen für die H-Atome konvergierte $2 \mathrm{u}$ einem konventionellen $\boldsymbol{R}$-Wert von 0.048 (0.054 inklusive 123 unbeobachteter Reflexe $\left.\left(\left|F_{c}\right|>k F_{0}\right)\right)$. Wegen Extinktion wurden zwei Reflexe aus der Verfeinerung herausgenommen. Die Atomkoordinaten stehen in Tab. 241). Sämtliche Rechnungen wurden im Rechenzentrum (IBM 370) der Universităt Heidelberg durchgefuhrt.

1) R. Huisgen, P. H. J. Ooms, M. Mingin und N. L. Allinger, J. Am. Chem. Soc. 102, 3951 (1980), und dort zit. Lit.

2) $R$. Huisgen, G. Szeimies und L. Mobbius, Chem. Ber. 100, 2494 (1967).

3) A. Eckell, R. Huisgen, R. Sustmann, G. Wallbillich, D. Grashey und E. Spindler, Chem. Ber. 100, 2192 (1967).

4) K. Bast, M. Christl, R. Huisgen und W. Mack, Chem. Ber. 106, 3312 (1973).

5) Kurzmitteilung: $M$. Christl, Angew. Chem. 85, 666 (1973); Angew. Chem., Int. Ed. Engl. 12, 660 (1973).

๑) Zusammenfassung uber Benzvalen: M. Christl, Angew. Chem. 93, 515 (1981); Angew. Chem., Int. Ed. Engl. 20, 529 (1981).

7) $R$. Huisgen und $W$. Mack, Chem. Ber. 105, 2805 (1972).

8) $R$. Huisgen und M. Christl, Chem. Ber. 106, 3291 (1973).

9) $R$. Sustmann, $R$. Huisgen und $H$. Huber, Chem. Ber. 100, 1802 (1967).

10) 10a) M. Christl, H.-J. Luddeke, A. Nagyrevi-Neppel und G. Freitag, Chem. Ber. 110, 3745 (1977). - 10b) M. Christl und R. Herbert, Chem. Ber. 112, 2022 (1979). - 10c) M. Christl, $H$. Leininger und $P$. Kemmer, Chem. Ber. 117, 2963 (1984).

11) C. Wentrup, B. Gerecht und H. Briehl, Angew. Chem. 91, 503 (1979); Angew. Chem., Int. Ed. Engl. 18, 467 (1979).

12) F. De Sarlo, A. Brandi, A. Guarna, A. Goti und S. Corezzi, Tetrahedron Lett. 24, 1815 (1983).

13) 13a) R. O. C. Norman, R. Purchase und C. B. Thomas, J. Chem. Soc., Perkin Trans. 1 1972, 1701. - 136) A. Vasella, Helv. Chim. Acta 60, 1273 (1977); S. Mzengeza und R. A. Whitney, J. Chem. Soc., Chem. Commun. 1984, 606.

14) $R$. Jacquier, J.-L. Olive, C. Petrus und F. Petrus, Tetrahedron Lett. 1975, 2337.

15) 15a) M. V. Kashutina, S. L. Ioffe und V. A. Tartakovskii, Dokl. Akad. Nauk SSSR 218, 109 (1974) [Chem. Abstr. 82, 43227q (1975)]. - 15b) $K$. Torssell und $O$. Zeuthen, Acta Chem. Scand., Ser. B 32, 118 (1978). - 15c) N. B. Das und K. B. G. Torssell, Tetrahedron 39, 2247 (1983).

16 P. A. Wade, H.-K. Yen, S. A. Hardinger, M. K. Pillay, N. V. Amin, P. D. Vail und S. D. Morrow, J. Org. Chem. 48, 1796 (1983).

17) $R$. Sustmann, Tetrahedron Lett. 1971, 2717.

18) P. Bischof, J. A. Hashmall, E. Heilbronner und V. Hornung, Helv. Chim. Acta 52, 1745 (1969).

19) R. Gleiter, Top. Curr. Chem. 86, 197 (1979).

20) $W$. Fliege und $R$. Huisgen, Liebigs Ann. Chem. 1973, 2038.

21) N. G. Rondan, M. N. Paddon-Row, P. Caramella, J. Mareda, P. H. Mueller und K. N. Houk, J. Am. Chem. Soc. 104, 4974 (1982).

22) J. Spanget-Larsen und $R$. Gleiter, Tetrahedron Lett. 23, 2435 (1982).

23) S. H. Andersen, N. B. Das, R. D. Jorgensen, G. Kjeldsen, J. S. Knudsen, S. C. Sharma und K. B. G. Torssell, Acta Chem. Scand., Ser. B 36, 1 (1982).

24) $U$. Stache, W. Fritsch und $H$. Ruschig, Liebigs Ann. Chem. 685, 228 (1965); G. W. Moersch, E. L. Wittle und W. A. Neuklis, J. Org. Chem. 32, 1387 (1967); J. Kalvoda und H. Kaufmann, J. Chem. Soc., Chem. Commun. 1976, 209; A. P. Kozikowski und M. Adamczyk, J. Org. Chem. 48, 366 (1983).

Chem. Ber. 119 (1986) 
25) $P$. A. Wade und H. R. Hinney, J. Am. Chem. Soc. 101, 1319 (1979); P. A. Wade und M. $K$. Pillay, J. Org. Chem. 46, 5425 (1981).

26) A. Brandi, F. De Sarlo, A. Guarna und G. Speroni, Synthesis 1982, 719; F. De Sarlo, A. Brandi, A. Goti, A. Guarna und P. Rovero, Heterocycles 20, 511 (1983).

27) $G$. Dittus in Methoden der organischen Chemie (Houben-Weyl-Müller), 4. Aufl., Bd. VI/3, S. 367, Thieme, Stuttgart 1965.

28) Wingfoot Corp. (Erf. P. H. Wise), US-Pat. 2473486 (14. Juni 1949) [Chem. Abstr. 43, 7953a (1949)].

29) N. J. Turro, C. A. Renner, T. J. Katz, K. B. Wiberg und H. A. Connon, Tetrahedron Lett. $1976,4133$.

30) U. Burger und G. Gandillon, Tetrahedron Lett. 1979, 4281; U. Burger, G. Gandillon und J. Mareda, Helv. Chim. Acta 64, 844 (1981).

31) A.-D. Schluter, J. Belzner, U. Heywang und G. Szeimies, Tetrahedron Lett. 24, 891 (1983); Dissertation A.-D. Schlüter, Univ. Munchen 1984.

32) T. J. Katz und K. C. Nicolaou, J. Am. Chem. Soc. 96, 1948 (1974).

33) T. J. Katz, E. J. Wang und N. Acton, J. Am. Chem. Soc. 93, 3782 (1971); T. J. Katz, R. J. Roth, N. Acton und E. J. Carnahan, Org. Synth. 53, 157 (1973).

34) 34a) $M$. Christl und $R$. Herbert, Org. Magn. Reson. 12, 150 (1979). - 34b) $M$. Christl und $S$. Freund, Chem. Ber, 118, 979 (1985).

35) $M$. Christl, R. Lang, W. Reimann und H. Irngartinger, Chem. Ber. 117, 959 (1984), dort weitere Zitate.

36) H. Irngartinger und K. L. Lukas, Angew. Chem. 91, 750 (1979); Angew. Chem., Int. Ed. Engl. 18, 694 (1979).

37 37a) M. N. Paddon-Row, K. N. Houk, P. Dowd, P. Garner und R. Schappert, Tetrahedron Lett. 22, 4799 (1981). - 37b) M. Eisenstein und F. L. Hirshfeld, Acta Crystallogr., Sect. B 39, 61 (1983). - 37e) P. G. Gassman, M. L. Greenlee, D. A. Dixon, S. Richtsmeier und J. Z. Gougoutas, J. Am. Chem. Soc. 105, 5865 (1983).

38) C. Grundmann und J. M. Dean, J. Org. Chem. 30, 2809 (1965).

39) A. Werner und H. Buss, Ber. Dtsch. Chem. Ges. 27, 2193 (1894); K.-C. Liu, B. R. Shelton und $R$. K. Howe, J. Org. Chem, 45, 3916 (1980).

40) J. P. Declercq, G. Germain, P. Main und M. M. Woolfson, Acta Crystallogr., Sect. A 29, 231 (1973).

41) Weitere Einzelheiten zur Kristallstrukturuntersuchung können beim Fachinformationszentrum Energie Physik Mathematik, D-7514 Eggenstein-Leopoldshafen, unter Angabe der Hinterlegungsnummer CSD 51 527, der Autoren und des Zeitschriftenzitats angefordert werden.

[174/85] 